PROCEEDINGS OF THE AMERICAN MATHEMATICAL SOCIETY

Volume 127, Number 7, Pages 2073-2081

S 0002-9939(99)04807-8

Article electronically published on February 26, 1999

\title{
ON THE DISJOINTNESS PROBLEM FOR GAUSSIAN AUTOMORPHISMS
}

\author{
M. LEMAŃCZYK AND F. PARREAU \\ (Communicated by Mary Rees)
}

\begin{abstract}
If $T_{\sigma_{1}}, T_{\sigma_{2}}$ are two Gaussian automorphisms, where $\sigma_{1}$ and $\sigma_{2}$ are concentrated on independent sets, then we have a dichotomy: either they are spectrally disjoint or they have a common factor.

As an application, we construct non-rigid automorphisms which are spectrally determined.
\end{abstract}

\section{INTRODUCTION}

The notion of disjointness between two automorphisms of a Lebesgue space has been introduced by Furstenberg in [3] as a concept of extremal non-isomorphism. It was already shown in [3] that if two automorphisms have a common nontrivial factor then they cannot be disjoint. There however exist automorphisms without common factors which are not disjoint (see Rudolph [13]). In certain classes of automorphisms the lack of common factors implies disjointness (e.g. [6]). We recall that spectral disjointness implies disjointness (e.g. [4]).

In this paper we study the problem of disjointness in some special classes of Gaussian automorphisms. We show that for two Gaussian-Kronecker automorphisms we have a dichotomy: either they are spectrally disjoint or they have a common factor. In fact, we prove that this dichotomy holds for Gaussian automorphisms whose spectral mesures are concentrated on independent sets. Such automorphisms have necessarily simple spectra and there are some of them which are mixing ([9], Ch. XIII, Theorem 3.8). In [8] it is shown however that the dichotomy is no longer valid in a larger setting.

The proof of the main result is self-contained, depending only on elementary properties of convolution powers of measures concentrated on independent sets (Section 3), and on basic constructions of group factors of Gaussian automorphisms.

As an application of our considerations we construct non-rigid automorphisms $T$ with the property that whenever an automorphism $T_{1}$ is spectrally isomorphic to $T$ then in fact these two automorphisms are measure-theoretically isomorphic.

We would like to thank J.- P. Thouvenot for fruitful discussions on the subject.

Received by the editors April 23, 1997 and, in revised form, October 9, 1997.

1991 Mathematics Subject Classification. Primary 28D05, 43A05.

The first author's research was partially supported by KBN grant 2 P301 03107 (1994).

(C)1999 American Mathematical Society 


\section{BASIC DEFINITIONS AND NOTATION}

Let $(X, \mathcal{B}, \mu)$ be a standard Borel space. On this space we consider the group of automorphisms $\operatorname{Aut}(X, \mathcal{B}, \mu)$ of measurable bijections $T: X \rightarrow X$ preserving $\mu$, endowed with the weak topology (in which $T_{n} \rightarrow T$ iff $\mu\left(T_{n} A \Delta T A\right)+$ $\mu\left(T_{n}^{-1} A \Delta T^{-1} A\right) \rightarrow 0$ for each $\left.A \in \mathcal{B}\right)$. Given $T \in \operatorname{Aut}(X, \mathcal{B}, \mu)$ we denote $C(T)$ the centralizer of $T$, i.e. the set of $S \in \operatorname{Aut}(X, \mathcal{B}, \mu)$ which commutes with $T$. With the weak topology $C(T)$ becomes a Polish group. We say that $T$ is rigid if there exists $n_{t} \rightarrow \infty$ such that $T^{n_{t}} \rightarrow I d$ weakly.

A $T$-invariant $\sigma$-algebra $\mathcal{A} \subset \mathcal{B}$ is called a factor of $(X, \mathcal{B}, \mu)$ (more precisely, the quotient action of $T$ on $(X, \mathcal{A}, \mu)$ is a factor of $T)$. A subset $\mathcal{F} \subset C(T)$ determines a factor

$$
\mathcal{A}(\mathcal{F})=\{A \in \mathcal{B}:(\forall S \in \mathcal{F}) S A=A\} .
$$

Note that $\mathcal{A}(\mathcal{F})=\mathcal{A}(\overline{G p(\mathcal{F})})$. A special role is played by compact subgroups of $C(T)$. In such a case, if $T$ is weakly mixing, the corresponding factor is never trivial.

Two automorphisms $T_{1}$ and $T_{2}$ are said to be disjoint [3] if whenever $T_{1}$ and $T_{2}$ both appear as factors of an ergodic automorphism $T$, then these factors are independent.

The circle group $\mathbf{T}$ will be treated as the group of complex numbers of unit modulus (except in Section 3). By a measure $\sigma$ on $\mathbf{T}$ we shall always mean a finite positive Borel measure. Its Fourier transform is defined by $\widehat{\sigma}[n]=\int z^{n} d \sigma(z)$, $n \in \mathbf{Z}$. We denote by $\tilde{\sigma}$ the measure defined by $\tilde{\sigma}(A)=\sigma(\bar{A}), A \in \mathcal{B}(\mathbf{T})$, and $\sigma$ is symmetric if $\tilde{\sigma}=\sigma$ or, equivalently, if its Fourier transform is real.

Each $T \in \operatorname{Aut}(X, \mathcal{B}, \mu)$ determines a unitary operator $U_{T}: L^{2}(X, \mathcal{B}, \mu) \rightarrow$ $L^{2}(X, \mathcal{B}, \mu), U_{T} f=f \circ T$. Given $f \in L^{2}(X, \mu)$, denote by $Z(f)$ the cyclic space generated by $f$ under $U_{T}$, that is, the closed span of $U_{T}^{n} f, n \in \mathbf{Z}$. The spectral measure of $f$ is the measure $\sigma_{f}$ on $\mathbf{T}$ given by

$$
\widehat{\sigma}_{f}[n]=\int_{X} z^{n} d \sigma_{f}(z)=\left(U_{T}^{n} f, f\right)=\int_{X}\left(f \circ T^{n}\right) \cdot \bar{f} d \mu .
$$

The spectral isomorphism $Z(f) \rightarrow L^{2}\left(\mathbf{T}, \sigma_{f}\right)$ maps $U_{T}^{n} f$ to $z^{n}, n \in \mathbf{Z}$. It yields a unitary equivalence between the restriction of $U_{T}$ to $Z(f)$ and the operator $V$ of multiplication by $z$ on $L^{2}\left(\mathbf{T}, \sigma_{f}\right)$.

There exists $f \in L^{2}(X, \mu)$ such that $\sigma_{f} \gg \sigma_{g}$ for every $g \in L^{2}(X, \mu)$. The type of $\sigma_{f}$ is then called the maximal spectral type of $U_{T}$. The operator $U_{T}$ is spectrally determined by its maximal spectral type $\sigma_{T}=\sigma_{f}$ and a function $M_{T}: \mathbf{T} \longrightarrow$ $\{1,2, \ldots\} \cup\{\infty\}$ (defined $\sigma_{T}$-a.e.) called the multiplicity function. For details, see $[12]$.

Two automorphisms $T_{1}$ and $T_{2}$ are called spectrally disjoint if $\sigma_{T_{1}}$ and $\sigma_{T_{2}}$ are mutually singular except for the point mass at 1 .

Put $X=\mathbf{R}^{\mathbf{Z}}, \mathcal{B}$ the $\sigma$-algebra of Borel sets and let $T: X \rightarrow X$ be the shift $(T x)_{n}=x_{n+1},(n \in \mathbf{Z})$. Let $f_{0}$ denote the 0 -coordinate projection.

A probability measure $\mu$ on $\mathcal{B}$ is called a Gaussian measure if the process $\left(f_{0} \circ T^{n}\right)$ is a stationary centered Gaussian process. The Gaussian dynamical system $(T, X, \mathcal{B}, \mu)$ is completely determined by the spectral measure $\sigma=\sigma_{f_{0}}$ of this process, given by

$$
\widehat{\sigma}[n]=\int_{X}\left(f_{0} \circ T^{n}\right) \cdot f_{0} d \mu .
$$


As $\widehat{\sigma}$ is real, $\sigma$ is symmetric. Conversely any symmetric measure $\sigma$ on $\mathbf{T}$ may be obtained as the spectral measure of a Gaussian process. We denote $\left(T_{\sigma}, X, \mathcal{B}, \mu_{\sigma}\right)$ the corresponding Gaussian dynamical system. Throughout, $\sigma$ is assumed to be continuous; equivalently $T_{\sigma}$ is weakly mixing.

Let $\sigma^{(n)}$ denote the $n$-th convolution power of $\sigma$, for $n \geq 1$. The maximal spectral type of $T_{\sigma}$ on $L_{0}^{2}\left(X, \mathcal{B}, \mu_{\sigma}\right)$, the orthocomplement of constant functions, is given by

$$
\exp ^{\prime} \sigma=\sum_{n=1}^{\infty} \frac{1}{n !} \sigma^{(n)} \text {. }
$$

The cyclic subspace $\mathcal{H}^{(1)}=Z\left(f_{0}\right)$ is called the first Wiener chaos of $T_{\sigma}$. If $g \in L^{2}(\mathbf{T}, \sigma),|g|=1$ a.e., then $g$ determines a unitary operator

$$
W_{g}: L^{2}(\mathbf{T}, \sigma) \longrightarrow L^{2}(\mathbf{T}, \sigma), \quad W_{g} f=g f,
$$

such that $W_{g} V=V W_{g}$ (in fact each unitary operator of $L^{2}(\mathbf{T}, \sigma)$ that commutes with $V$ is of the form $W_{g}$ ). By the spectral isomorphism, $W_{g}$ defines an operator of $\mathcal{H}^{(1)}$ which commutes with $U_{T}$. If in addition $g(\bar{z})=\overline{g(z)}$ a.e., then as noticed in [7], this operator extends in a unique way to an element $S_{g}$ of $C\left(T_{\sigma}\right)$.

Put

$$
\mathcal{F}_{\sigma}=\left\{g \in L^{2}(\mathbf{T}, \sigma):|g|=1, g(\bar{z})=\overline{g(z)} \sigma \text {-a.e. }\right\} \text {. }
$$

On $\mathcal{F}_{\sigma}$ we consider the inherited $L^{2}(\sigma)$-topology $\left(\mathcal{F}_{\sigma}\right.$ is a closed subset of $\left.L^{2}(\sigma)\right)$ and with the pointwise multiplication $\mathcal{F}_{\sigma}$ becomes a Polish group. Then, the group $\mathcal{F}_{\sigma}$ can be viewed as a subgroup of the centralizer of $T_{\sigma}$. In certain situations, for example if $\sigma \perp \sigma^{(n)}$ for each $n \geq 2, \mathcal{F}_{\sigma}$ can be identified with $C\left(T_{\sigma}\right)$.

Now, if $\mathcal{K} \subset \mathcal{F}_{\sigma}$ is a compact subgroup, it gives rise to a compact subgroup (still denoted by $\mathcal{K}$ ) of $C\left(T_{\sigma}\right)$ which in turn determines a factor $\mathcal{A}(\mathcal{K})$ of $T_{\sigma}$, defined by (1). When $\mathcal{K}=\{-1,1\}$, the corresponding factor is called the even factor of $T_{\sigma}$ (see $[11])$.

Besides $\mathcal{F}_{\sigma}$ does not contain any proper cocompact subgroup. Indeed, it is equivalent to saying that $\mathcal{F}_{\sigma}$ does not admit a non-trivial character. For $n \geq 1$, let $\mathcal{F}_{\sigma}^{(n)}=\left\{g \in \mathcal{F}_{\sigma}: g^{n}=1 \sigma\right.$ - a.e. $\}$. Given $g \in \mathcal{F}_{\sigma}^{(n)}$ and $t \in[0,1]$, let $g_{t}(z)=g(z)$ if $z=e^{2 \pi i s}$ for some $s \in(-t / 2, t / 2)$ and $g_{t}(z)=1$ otherwise. This yields an arc connecting 1 with $g$ in $\mathcal{F}_{\sigma}^{(n)}$. In particular, $\mathcal{F}_{\sigma}^{(n)}$ is connected. Now, each character of $\mathcal{F}_{\sigma}$ takes only finitely many values on $\mathcal{F}_{\sigma}^{(n)}$ and thus must be trivial on $\mathcal{F}_{\sigma}^{(n)}$. Since $\bigcup_{n} \mathcal{F}_{\sigma}^{(n)}$ is dense in $\mathcal{F}_{\sigma}$, the same holds true for $\mathcal{F}_{\sigma}$.

\section{Some Remarks on Gaussian-KroneCKer Automorphisms}

A compact subset $K$ of $\mathbf{T}$ is called a Kronecker set if for each continuous function $f: K \rightarrow \mathbf{T}$ there exists a sequence $\left(\chi_{k}\right)$, where $\chi_{k}(z)=z^{n_{k}}$, of characters of $\mathbf{T}$ such that $\chi_{k} \rightarrow f$ uniformly on $K$. A Kronecker set is an example of an independent set: a Borel set $L$ in $\mathbf{T}$ is called independent if it admits only trivial arithmetic relations between its elements $\left(z_{1}^{n_{1}} \cdot \ldots \cdot z_{k}^{n_{k}}=1\right.$ iff $\left.n_{1}=\cdots=n_{k}=0\right)$.

A continuous measure $\sigma$ on $\mathbf{T}$ is said to be a Kronecker measure if for each $f \in$ $L^{2}(\mathbf{T}, \sigma)$ of modulus 1 there exists a sequence $\left(\chi_{k}\right)$ of characters which converges to $f$ in $L^{2}(\mathbf{T}, \sigma)$.

Lemma 1. A continuous measure $\sigma$ on $\mathbf{T}$ is a Kronecker measure if and only if for every $\varepsilon>0$ there exists a Kronecker set $K_{\varepsilon}$ with $\sigma\left(K_{\varepsilon}\right)>1-\varepsilon$. Moreover, in this case, $\sigma$ is concentrated on an independent Borel set. 
Proof. The "if" part is immediate. Conversely, suppose that $\sigma$ is a Kronecker measure. Let $\left\{f_{m}\right\}$ be a countable dense family in $C(\mathbf{T}, \mathbf{T})$ and choose for every $m$ a sequence $\left(\chi_{i}^{(m)}\right)$ of characters which converges to $f_{m}$ in $L^{2}(\mathbf{T}, \sigma)$. There exists for every $m \geq 1$ a compact set $K_{m} \subset \mathbf{T}$ such that $\sigma\left(K_{m}^{c}\right)<1 / 2^{m+1}$ and some subsequence of $\left(\chi_{i}^{(m)}\right)$ converges to $f_{m}$ uniformly on $K_{m}$. Letting $L_{n}=\bigcap_{m>n} K_{m}$, $(n \geq 1)$ we obtain that $\sigma\left(L_{n}^{c}\right)<1 / 2^{n}$, and each $f_{m}$ is uniformly approximated by characters on $K$. Since the family $\left\{\left.f_{m}\right|_{L_{n}}: m \geq n\right\}$ of restrictions of $f_{m}$ to $L_{n}$ forms a dense subset of $C\left(L_{n}, \mathbf{T}\right)$, it follows that $L_{n}$ is a Kronecker set.

Now, the second part of the proposition follows from the fact that $\sigma$ is concentrated on $\bigcup_{n} L_{n}$, and that an increasing union of independent sets is independent.

By classical theory of Gaussian automorphisms ([1], pp. 373-374), we know that if $\sigma$ is concentrated on $K \cup \bar{K}$ where $K$ is an independent Borel set then $T_{\sigma}$ has simple spectrum.

We say that $\sigma$ is a symmetric Kronecker measure if $\sigma=\frac{1}{2}\left(\sigma_{0}+\tilde{\sigma}_{0}\right)$, where $\sigma_{0}$ is a Kronecker measure. This is equivalent to saying that the characters are dense in $\mathcal{F}_{\sigma}$. Also, $\sigma$ is a symmetric Kronecker measure if and only if $C\left(T_{\sigma}\right)=\overline{\left\{T_{\sigma}^{n}: n \in \mathbf{Z}\right\}}$, i.e. if $T_{\sigma}$ satisfies the Weak Closure Theorem.

Let $g \in \mathcal{F}_{\sigma}$. By $g_{*} \sigma$, we mean the image measure of $\sigma$ by $g$, i.e.

$$
g_{*} \sigma(A)=\sigma\left(g^{-1} A\right) \quad(A \in \mathcal{B}(\mathbf{T})) .
$$

Proposition 1. Let $\sigma$ be a symmetric Kronecker measure. Assume that $h \in \mathcal{F}_{\sigma}$ and that the closed subgroup generated by $g(z)=z^{-1} h(z)$ is compact. Then $h$ generates a dense subgroup of $\mathcal{F}_{\sigma}$ and $h_{*} \sigma$ is a symmetric Kronecker measure.

Proof. Let $\left(g_{n}\right)$ be any sequence in $\mathcal{F}_{\sigma}$. Choose $\left(k_{n}\right)$ so that $g_{n}(z) z^{k_{n}} \rightarrow 1$ in $\mathcal{F}_{\sigma}$; since the closed subgroup generated by $g$ is compact, by taking a subsequence, we can assume that $\left(g^{k_{n}}\right)$ converges. Then $\left(g_{n} h^{k_{n}}\right)$ converges. This proves that the closed subgroup $\mathcal{J}$ generated by $h$ is cocompact. Since $\mathcal{F}_{\sigma}$ has no cocompact proper subgroups, $\mathcal{J}$ is equal to $\mathcal{F}_{\sigma}$. Now $f \mapsto f \circ h$ yields an embedding of $\mathcal{F}_{h_{*} \sigma}$ into $\mathcal{F}_{\sigma}$, so the density of $\left\{z^{n}: n \in \mathbf{Z}\right\}$ in $\mathcal{F}_{h_{*} \sigma}$ follows from the density of $\left\{h^{n}: n \in \mathbf{Z}\right\}$ in $\mathcal{F}_{\sigma}$.

Remark 1. Suppose that $g \in \mathcal{F}_{\sigma}$ takes countably many values, i.e., $\sigma=\sum_{i>1} \sigma_{i}$ where $\sigma_{i} \perp \sigma_{j}$ whenever $i \neq j$ and $g(z)=z_{i} \sigma_{i}$-a.e., $i \geq 1$. Then the closed subgroup generated by $g$ is compact, whence $h_{*} \sigma=\sum \delta_{z_{i}} * \sigma_{i}$ is a symmetric Kronecker measure. A similar statement holds for the non-symmetric case. In particular any translation of a Kronecker measure is a Kronecker measure.

We shall say that a symmetric measure $\sigma$ on $\mathbf{T}$ has the Foias-Stratila (FS) property if for each ergodic automorphism $T:(X, \mathcal{B}, \mu) \rightarrow(X, \mathcal{B}, \mu)$, any real valued $f \in L_{0}^{2}(X, \mathcal{B}, \mu)$ such that $\sigma_{f}=\sigma$ we have that $f$ is a real Gaussian variable: Foias and Stratila ([2] or [1], pp. 375-379) proved this property for measures concentrated on Kronecker sets. By small changes in the first part of the proof (or directly from this theorem, Lemma 1 and the elementary fact that given a sequence of measures $\sigma_{n} \leq \sigma_{f}$ which converge to $\sigma_{f}$ in the variation norm we can find a sequence of real functions $f_{n}$ in $Z(f)$, with spectral measures $\sigma_{n}$, which converge to $f$ in $L^{2}$ ), we obtain 
Theorem 1 (Foias-Stratila). If $\sigma$ is a symmetric Kronecker measure, then $\sigma$ has FS property.

Proposition 2. If $\sigma$ has FS property and $\sigma^{\prime} \ll \sigma$ is symmetric then $\sigma^{\prime}$ has FS property.

Proof. The same elementary argument as above allows us to restrict ourselves to the case $\sigma^{\prime} \leq \sigma$; given an ergodic automorphism $T:(X, \mathcal{B}, \mu) \rightarrow(X, \mathcal{B}, \mu)$ take a real valued $f \in L_{0}^{2}(X, \mathcal{B}, \mu)$ whose spectral measure equals $\sigma^{\prime}$. Write $\sigma=\sigma^{\prime}+\sigma_{1}$ and consider $T \times T_{\sigma_{1}}$. This automorphism is still ergodic and it is clear that if a real function $g$ in the first chaos of $T_{\sigma_{1}}$ has the spectral measure $\sigma_{1}$ then the spectral measure of

$$
F(x, y)=f(x)+g(y) \in L^{2}\left(X \times \mathbf{R}^{\mathbf{Z}}, \mu \times \mu_{\sigma_{1}}\right)
$$

is equal to $\sigma^{\prime}+\sigma_{1}=\sigma$. Hence $F$ is a Gaussian variable. Since $f$ and $g$ are independent, $f$ must be a Gaussian variable.

Lemma 2. Let $\sigma_{1}$ and $\sigma_{2}$ be symmetric measures with FS property. Suppose that $T_{\sigma_{1}}$ and $T_{\sigma_{2}}$ are disjoint. Then $\sigma=\sigma_{1}+\sigma_{2}$ has FS property.

Proof. Let $T:(X, \mathcal{B}, \mu) \longrightarrow(X, \mathcal{B}, \mu)$ be an ergodic automorphism and let $f \in$ $L_{0}^{2}(X, \mathcal{B}, \mu)$ be real valued with $\sigma_{f}=\sigma$. By the hypothesis we must have $\sigma_{1} \perp \sigma_{2}$ and thus there is a unique decomposition $f=f_{1}+f_{2}$ where $\sigma_{f_{1}}=\sigma_{1}$ and $\sigma_{f_{2}}=\sigma_{2}$. Since $\sigma_{j}$ is symmetric, $\sigma_{\bar{f}_{j}}=\sigma_{j}$ for $j=1,2$ and, by unicity of the decomposition, it follows that the $f_{j}$ are real functions. Denote $\mathcal{B}_{j}$ the $T$-invariant $\sigma$-algebra generated by $f_{j}$. By our assumptions, each $f_{j}$ is a Gaussian variable, so $\left.T\right|_{\mathcal{B}_{j}}$ is isomorphic to $T_{\sigma_{j}}$. Now, since $T_{\sigma_{1}}$ and $T_{\sigma_{2}}$ are disjoint, the factors are independent and hence $f$ is a Gaussian variable as a sum of independent Gaussian variables.

\section{On THE ARITHMETIC OF CONVOLUTIONS OF MEASURES CONCENTRATED ON INDEPENDENT SETS}

In this section the circle group will be treated additively, i.e. $\mathbf{T}=\mathbf{R} / \mathbf{Z}$.

Theorem 2. Let $\sigma, \tau$ and $\rho$ be continuous measures on $\mathbf{T}$. Assume that $\sigma$ is symmetric and concentrated on $K \cup(-K)$, where $K$ is an independent Borel set. Suppose moreover that, for some positive integer $n$, the convolutions $\rho * \tau$ and $\sigma^{(n)}$ are not mutually singular. Then there exists a positive integer $l \leq n$ such that $\tau$ and a certain translation of $\sigma^{(l)}$ are not mutually singular.

Before proving this theorem, we need some notation and two lemmas. Let $K_{1}=$ $K \cup(-K)$; for $l \geq 1$, denote $K_{l}=\underbrace{K_{1}+\ldots+K_{1}}_{l}$ and $\pi_{l}: K_{1}^{l} \rightarrow K_{l},\left(x_{1}, \ldots, x_{l}\right) \mapsto$ $x_{1}+\ldots+x_{l}$. Since $\sigma$ is continuousm, the product measure $\sigma^{l}$ on $K_{1}^{l}$ gives 0 measure to each of the subsets of $K_{1}^{l}$ where one coordinate is fixed, and to the subset where two coordinates are opposite. By independence, it follows that $\sigma^{(l)}\left(K_{l-1}+x\right)=0$ for every $x \in K_{1}$ and $\sigma^{(l)}\left(K_{l-2}\right)=0$ (for the sake of completeness, let $K_{0}=\{0\}$ and $K_{l}=\emptyset$ for $\left.l<0\right)$.

Let us say that a measure $\mu$ concentrated on $K_{l}$ which shares this property is properly concentrated on $K_{l}$.

Lemma 3. Let $\sigma, \tau$ and $\rho$ satisfy the assumptions of Theorem 2 and suppose moreover that $\rho$ is concentrated on $K_{m}$ and $\tau$ is properly concentrated on $K_{l}$, where $m$ and $l$ are positive integers. Then $\tau$ and $\sigma^{(l)}$ are not mutually singular. 
Proof. Notice first that we may also assume $\rho\left(K_{m-2}\right)=0$. Indeed, let $\rho^{\prime}=\left.\rho\right|_{K_{m-2}}$; if $\rho^{\prime} * \tau \perp \sigma^{(n)}$, we can substitute $\rho-\rho^{\prime}$ for $\rho$ and otherwise we can substitute $\rho^{\prime}$ for $\rho$ and $m-2$ for $m$.

We have

$$
\int_{\mathbf{T}} \tau\left(K_{n}-y\right) d \rho(y)=\rho * \tau\left(K_{n}\right)>0 .
$$

Since $\rho\left(K_{m-2}\right)=0$, we can find $y_{1}, \ldots, y_{m}$ in $K_{1}$ such that no cancellation occurs in the sum $y=y_{1}+\cdots+y_{m}$ and $\tau\left(K_{n}-y\right)>0$. Then, since $\tau$ is properly concentrated on $K_{l}$,

$$
\left(K_{n}-y \cap K_{l}\right) \backslash\left(K_{l-2} \cup K_{l-1}-y_{1} \cup \cdots \cup K_{l-1}-y_{m}\right)
$$

is not empty. Thus, there exist $x_{1}, \ldots, x_{l}$ in $K_{1}$ such that

$$
x_{1}+\cdots+x_{l}+y_{1}+\cdots+y_{m} \in K_{n}
$$

and no cancellation occurs in this sum. This proves $l+m \leq n$.

On the other hand $\rho * \tau$ is concentrated on $K_{m}+K_{l} \subset K_{m+l}$ and $\sigma^{(n)}$ is properly concentrated on $K_{n}$, which implies $l+m \geq n$ and thus $l+m=n$.

Suppose now that there is a Borel set $B$ in $K_{l}$ such that $\tau$ is concentrated on $B$ and $\sigma^{(l)}(B)=0$. Then $\rho * \tau$ is concentrated on $K_{m}+B$, and $\sigma^{l}\left(B^{\prime}\right)=0$, where $B^{\prime}=\pi_{l}^{-1}(B)$. It follows $\sigma^{n}\left(K_{1}^{m} \times B^{\prime}\right)=0$ and the same is true for each set obtained from $K_{1}^{m} \times B^{\prime}$ by permutation of coordinates. The union of these sets contains the preimage of $\left(K_{m}+B\right) \backslash\left(K_{n-2}\right)$ in $K_{1}^{n}$, so $\sigma^{(n)}\left(K_{m}+B\right)=0$. This is a contradiction which shows that $\tau$ and $\sigma^{(l)}$ are not mutually singular.

The next lemma makes use of techniques developed in [5].

Lemma 4. Let $A$ be a Borel subset of $\mathbf{T}$. Then each finite positive Borel measure $\tau$ on $\mathbf{T}$ admits a decomposition (finite or countable)

$$
\tau=\sum_{j} \delta_{x_{j}} * \tau_{j}+\tau^{\prime}
$$

where $\tau_{j}$ is concentrated on $A$ and $\tau^{\prime}(A+x)=0$ for all $x \in \mathbf{T}$. Moreover, $\mu * \tau^{\prime}(A)=$ 0 for each finite Borel measure on $\mathbf{T}$.

Proof. Let $B$ be a Borel set of maximal measure (for $\tau$ ) among the countable unions of translations of $A$ (if $\mathcal{R}=\left\{C=C\left(x_{1}, x_{2}, \ldots\right.\right.$ ) : $\left.\left\{x_{1}, x_{2}, \ldots\right\} \subset \mathbf{T}\right\}$, where $C\left(x_{1}, x_{2}, \ldots\right)=\bigcup_{j=1}^{\infty} A+x_{j}$ and $\alpha=\sup _{C \in \mathcal{R}} \tau(C)$, then there is a sequence $C_{n} \in \mathcal{R}$ with $\tau\left(C_{n}\right) \longrightarrow \alpha$ and the set $\bigcup C_{n} \in \mathcal{R}$ will do). The restriction of $\tau$ to $B$ can be then represented as $\sum_{j} \delta_{x_{j}} * \tau_{j}$ and the restriction of $\tau$ to $\mathbf{T} \backslash B$ is equal to zero for all translations of $A$. Since

$$
\mu * \tau^{\prime}(A)=\int \tau^{\prime}(A-x) d \mu(x),
$$

the result follows.

Proof of Theorem 2. Applying Lemma 4 to $A=K_{1}$ and $\tau$ we find a decomposition $\tau=\sum_{j} \delta_{x_{j}^{(1)}} * \tau_{j}^{(1)}+\tau_{1}$; then we apply once more Lemma 4 to $A=K_{2}$ and $\tau_{1}$, obtaining

$$
\tau=\sum_{j} \delta_{x_{j}^{(1)}} * \tau_{j}^{(1)}+\sum_{j} \delta_{x_{j}^{(2)}} * \tau_{j}^{(2)}+\tau_{2} .
$$


We continue this procedure $n$ times. We finally obtain a decomposition $\tau=\sum_{j} \delta_{x_{j}} *$ $\tau_{j}+\tau^{\prime}$, where $\mu * \tau^{\prime}\left(K_{n}\right)=0$ for each finite Borel measure $\mu$ on $\mathbf{T}$ and for each $j$ there exists some $l_{j} \leq n$ such that $\tau_{j}$ is properly concentrated on $K_{l_{j}}$.

Let $\rho=\sum_{k} \delta_{y_{k}} * \rho_{k}+\rho^{\prime}$ be a similar decomposition of $\rho$, where $\rho_{k}$ is properly concentrated on $K_{m_{k}}$. We have $\delta_{y_{k}} * \rho_{k} * \delta_{x_{j}} * \tau_{j} \not \perp \sigma^{(n)}$ for some $j$ and $k$, whence

$$
\tilde{\rho}_{k} * \tilde{\tau}_{j} * \rho_{k} * \tau_{j} \not \perp \sigma^{(2 n)}
$$

since $\sigma$ is symmetric.

Now, $\tilde{\rho}_{k} * \tilde{\tau}_{j} * \rho_{k}$ is concentrated on $K_{2 m_{k}+l_{j}}$ and we can apply Lemma 3 to the measures $\sigma, \tau_{j}$ and $\tilde{\rho}_{k} * \tilde{\tau}_{j} * \rho_{k}$ (and $2 n$ instead of $n$ ). This yields $\tau_{j} \not \perp \sigma^{\left(l_{j}\right)}$ and finally $\tau \not \perp \delta_{x_{j}} * \sigma^{\left(l_{j}\right)}$.

Corollary 1. Let $\sigma$ and $\tau$ be finite positive, symmetric continuous Borel measures on $\mathbf{T}$. Assume that $\sigma$ is concentrated on $K \cup(-K)$, where $K$ is an independent Borel set and that $\tau$ is concentrated on a countable union of independent Borel sets.

Then either each convolution power of $\tau$ is singular with respect to every convolution power of $\sigma$, or $\tau$ and some translation of $\sigma$ are not mutually singular.

Proof. Suppose that $\tau^{(m)} \not \perp \sigma^{(n)}$ for some positive integers $m$ and $n$. If $m>1$, it follows from Theorem 2 with $\rho=\tau^{(m-1)}$ that there exist an integer $l \leq n$ and $x \in \mathbf{T}$ such that $\tau \not \perp \delta_{x} * \sigma^{(l)}$; if $m=1$, let $x=0$ and $l=n$.

Now, if $l=1$, the proof is complete. Otherwise, by the hypothesis on $\tau$, there exists an independent Borel set $L$ such that $\left.\tau\right|_{L \cup(-L)} \not \perp \delta_{x} * \sigma^{(l)}$. Then, Theorem 2 with $\tau^{\prime}=\left.\tau\right|_{L \cup(-L)}, \sigma$ and $\delta_{x} * \sigma^{(l-1)}$ in the roles of $\sigma, \tau$ and $\rho$, respectively, and $n=1$, shows that $\sigma$ is not singular with respect to some translation of $\tau^{\prime}$, hence of $\tau$.

\section{ON THE DISJOINTNESS PROBLEM OF GAUSSIAN AUTOMORPHISMS}

Lemma 5. Let $\sigma$ be a symmetric continuous measure on $\mathbf{T}$ and $h \in \mathcal{F}_{\sigma}$. If $h$ is one-to-one, then the action of $S_{h}$ on $\left(X, \mathcal{B}, \mu_{\sigma}\right)$ is isomorphic to $T_{h_{*} \sigma}$. If moreover the closed subgroup generated by $g(z)=z^{-1} h(z)$ is compact, then $T_{\sigma}$ and $T_{h_{*} \sigma}$ have a non-trivial common factor.

Proof. Directly from the definition (Section 1), the automorphism $S_{h}$ preserves the Gaussian space $\mathcal{H}^{(1)}$ of $L^{2}\left(X, \mu_{\sigma}\right)$ and its action is conjugate to the operator of multiplication by $h$ on $L^{2}(\mathbf{T}, \sigma)$ via the spectral isomorphism. Thus $\left(f_{0} \circ S_{h}^{n}\right)$ is a Gaussian process and its spectral measure $\tau$ is given by

$$
\widehat{\tau}[n]=\int_{X}\left(f_{0} \circ S_{h}^{n}\right) \cdot f_{0} d \mu_{\sigma}=\int_{\mathbf{T}} h^{n} d \sigma=\int_{\mathbf{T}} z^{n} d h_{*} \sigma(z)=\widehat{h_{*} \sigma}[n] .
$$

So, $\tau=h_{*} \sigma$ and $T_{\tau}$ appears as a factor of $S_{h}$. If $h$ is one-to-one, the family $\left\{h^{n}: n \in \mathbf{Z}\right\}$, spans linearly $L^{2}(\mathbf{T}, \sigma)$, whence $\left\{S_{h}^{n} f_{0}\right\}$ spans linearly $\mathcal{H}^{(1)}$, which in turn generates the whole $\sigma$-algebra: then $S_{h}$ is isomorphic to $T_{\tau}$.

Now, assume that the closed subgroup $\mathcal{K}$ of $\mathcal{F}_{\sigma}$ generated by $g(z)=z^{-1} h(z)$ is compact. Then clearly the identity map establishes an isomorphism of $T_{\sigma}$ and $S_{h}$ restricted to the corresponding factor

$$
\mathcal{A}(\mathcal{K})=\left\{A \in \mathcal{B}: S_{g} A=T_{\sigma}^{-1} S_{h} A=A\right\} .
$$

Moreover $\mathcal{A}(\mathcal{K})$ cannot be trivial since $T_{\sigma}$ is weakly mixing. 
Proposition 3. Let $\sigma$ and $\tau$ be two symmetric continuous measures on $\mathbf{T}$. If $\tau$ and some translation of $\sigma$ are not mutually singular, then $T_{\sigma}$ and $T_{\tau}$ have a non-trivial common factor.

Proof. Suppose $\delta_{z_{0}} * \sigma \not \perp \tau$, where $z_{0} \in \mathbf{T}$. We can find a positive measure $\eta$ such that

$$
\sigma^{\prime}=\frac{1}{2}(\eta+\tilde{\eta}) \ll \sigma \quad \text { and } \quad \tau^{\prime}=\frac{1}{2}\left(\delta_{z_{0}} * \eta+\delta_{\overline{z_{0}}} * \tilde{\eta}\right) \ll \tau .
$$

Since $T_{\sigma^{\prime}}$ is a factor of $T_{\sigma}$ and $T_{\tau^{\prime}}$ is a factor of $T_{\tau}$, it is enough to prove that $T_{\sigma^{\prime}}$ and $T_{\tau^{\prime}}$ have a common factor. By restricting $\eta$ to a Borel set $B$ of positive measure such that

$$
B \cap \bar{B}=\emptyset, \quad z_{0} B \cap \bar{z}_{0} \bar{B}=\emptyset
$$

we may furthermore assume that $\eta \perp \tilde{\eta}$ and that the function $h$ in $\mathcal{F}_{\sigma^{\prime}}$ defined by

$$
h(z)=\left\{\begin{array}{cc}
z_{0} z & \eta \text {-a.e. } \\
\bar{z}_{0} z & \tilde{\eta} \text {-a.e. }
\end{array}\right.
$$

is one-to-one. Notice that $h_{*} \sigma^{\prime}=\tau^{\prime}$. Since $g(z)=z^{-1} h(z)$ takes only two values, $g$ generates a compact subgroup in $\mathcal{F}_{\sigma^{\prime}}$. So, we can apply Lemma 5 , which completes the proof.

Now, applying Corollary 1 and Proposition 3, we get:

Theorem 3. Let $\sigma, \tau$ be two symmetric continuous measures on the circle. We assume that $\sigma$ is concentrated on $K \cup \bar{K}$ where $K$ is an independent Borel set and that $\tau$ is concentrated on a countable union of independent sets. Then either $T_{\sigma}$ and $T_{\tau}$ are spectrally disjoint or they have a common factor.

In particular, the assertion holds if both measures are Kronecker measures.

As an application, we obtain other measures $\sigma$ with the FS property. More precisely:

Theorem 4. Let $\sigma=\sum_{j \geq 1} \sigma_{j}$ where each $\sigma_{j}$ is a symmetric Kronecker measure. If for all $j \neq k, \sigma_{j}$ and any translation of $\sigma_{k}$ are mutually singular, then $\sigma$ has FS property.

Proof. It is sufficient to prove that every finite sum $\sigma_{n}^{\prime}=\sigma_{1}+\cdots+\sigma_{n}$ has FS property. By Corollary $1, T_{\sigma_{n+1}}$ and $T_{\sigma_{n}^{\prime}}$ are spectrally disjoint. So, the result follows from Lemma 2 by induction.

For each symmetric Kronecker measure $\sigma$ there exists a sequence $n_{i} \longrightarrow \infty$ such that $z^{n_{i}} \longrightarrow 1$ in $\mathcal{F}_{\sigma}$. Then $T_{\sigma}^{n_{i}} \longrightarrow$ Id weakly, so $T_{\sigma}$ is a rigid automorphism.

Theorem 4 enables us to construct non-rigid Gaussian automorphisms with FS property. Now we refer the reader to [9], Ch. XIII, $\S 3$, for the following result.

For each $N$ we can find $N$ disjoint Kronecker sets $K_{1}, \ldots, K_{N}$ whose union $K$ is independent and carries a continuous probability measure $\rho$ for which

$$
\limsup _{n \rightarrow \infty}|\widehat{\rho}[n]|=\frac{1}{N}
$$

Now, take $\sigma=\frac{1}{2}(\rho+\tilde{\rho})$ and put $\sigma_{j}$ to be equal to the restriction of $\sigma$ to $K_{j} \cup \overline{K_{j}}$. Since $K$ is independent, for all $j \neq k, \sigma_{j}$ and any translation of $\sigma_{k}$ are mutually singular and hence the assumptions of Theorem 4 are satisfied. Besides,

$$
|\widehat{\sigma}[n]|=|\operatorname{Re} \widehat{\sigma}[n]| \leq|\widehat{\rho}[n]| .
$$


Remark 2. Since the measure above is concentrated on an independent set, the associated Gaussian automorphism has simple spectrum. Moreover $\sigma$ has FS property and it follows that given an automorphism $T$ with $U_{T}$ and $U_{T_{\sigma}}$ spectrally isomorphic, we have $T$ and $T_{\sigma}$ measure-theoretically isomorphic. Hence, this example gives rise to a non-rigid example of automorphism which is spectrally determined.

We recall that it is still an open problem whether there exists a mixing Gaussian automorphism with the FS property.

Note also that Theorem 4 allows us to obtain measures which satisfy FS property without assuming a priori that they are concentrated on some independent set. By [9] (Ch. X, Lemma 2.10), there exist two Kronecker sets $K_{1}, K_{2} \subset \mathbf{T}$ such that $K_{1}+K_{2}=\mathbf{T}$. If we could construct two Kronecker measures $\sigma_{1}, \sigma_{2}$ such that $\sigma_{1} \perp \sigma_{2} * \delta_{x}$ (for all $x \in \mathbf{T}$ ) and $\sigma_{1} * \sigma_{2}$ non-purely singular then we could even construct automorphisms with non-singular spectra and FS property.

\section{REFERENCES}

[1] I.P. Cornfeld, S.W. Fomin, J.G. Sinai, Ergodic Theory, Springer-Verlag 1982. MR 87f:28019

[2] C. Foias, S. Stratila, Ensembles de Kronecker dans la théorie ergodique, C.R. Acad. Sci. Paris 267, 20A (1967), 166-168. MR 38:1234

[3] H. Furstenberg, Disjointness in ergodic theory, minimal sets and Diophantine approximation, Math. Syst. Theory 1 (1967), 1-49. MR 35:4369

[4] F. Hahn, W. Parry, Some characteristic properties of dynamical systems with quasi-discrete spectrum, Math. Syst. Theory 2 (1968), 179-198. MR 37:6435

[5] B. Host, F. Parreau, Sur une notion de pureté pour les mesures, C.R. Acad. Sci. Paris, t. 306, Série I (1998), 409-412. MR 89a:43001

[6] A. del Junco, D. Rudolph, On ergodic actions whose self-joinings are graphs, Erg. Th. Dyn. Syst. 7 (1988), 531-557. MR 89e:28029

[7] M. Lemańczyk, J. Sam Lazaro, Spectral analysis of certain compact factors for Gaussian dynamical systems, Isr. J. Math. 98 (1997), 307-328. CMP 97:15

[8] M. Lemańczyk, F. Parreau, J.- P. Thouvenot, Gaussian automorphisms whose ergodic selfjoinings are Gaussian, preprint.

[9] L. Lindahl, F. Paulsen, Thin Sets in Harmonic Analysis, Marcel Dekker (1971). MR 52: 14800

[10] D. Newton, On Gaussian processes with simple spectrum, Z. für Wahr. verw. Geb. 5 (1966), 207-209. MR 34:868

[11] D. Newton, W. Parry, On a factor automorphism of a normal dynamical system, Ann. Math. Stat. 37 (1966), 1528-1533. MR 34:6028

[12] W. Parry, Topics in Ergodic Theory, Cambridge Univ. Press 1981. MR 83a:28018

[13] D. J. Rudolph, An example of a measure-preserving map with minimal self-joinings and applications, J. Anal. Math. 35 (1979), 97-122. Proc. Erg. Th. Rel. Topics.II, 195-198, Georgenthal 1986 joinings in London Math. MR 81e:28011

Department of Mathematics and Computer Science, Nicholas Copernicus University, ul. Chopina 12/18, 87-100 Toruń, Poland

E-mail address: mlem@mat.uni.torun.pl

Laboratoire d'Analyse, Géométrie et Applications, URA CNRS 742, Université Paris 13, Av. J.-B. Clément, 93430 Villetaneuse, France

E-mail address: parreau@math.univ-paris13.fr 